\title{
A Generalized Error Distribution Copula-based method for portfolios risk assessment
}

\author{
Roy Cerqueti*, Massimiliano Giacalone ${ }^{\natural}$, Demetrio Panarello ${ }^{\sharp}$ \\ b University of Macerata, Department of Economics and Law. \\ Via Crescimbeni, 14. I-62100, Macerata, Italy. \\ Tel.: +39 0733 2583246; fax: +390733 2583205. Email: roy.cerqueti@unimc.it \\ Ł University of Naples 'Federico II', Department of Economics and Statistics. \\ Email: massimiliano.giacalone@unina.it \\ \# Parthenope University of Naples, Department of Economic and Legal Studies. \\ Email: demetrio.panarello@uniparthenope.it
}

January 17, 2019

\begin{abstract}
In this paper, we deal with the evaluation of Conditional Value-at-Risk in the framework of portfolio theory by using a modified Gaussian Copula - where the modification is obtained by introducing the Generalized Correlation Coefficient - and by assuming a Generalized Error Distribution with properly estimated shape parameter $p$ for the returns of the considered risky assets. In so doing, we add to the connection between standard Copula theory and financial risk assessment. A comparison analysis of our findings with those obtainable through a standard Gaussian Copula-based procedure in a set of real data is also presented.
\end{abstract}

Keywords: Econophysics, Portfolio theory, Conditional Value-at-Risk, Gaussian Copula, Generalized Error Distribution, Generalized Correlation Coefficient.

JEL Codes: G11, C16.

\section{Introduction}

One of the most important issues in finance is to correctly measure the risk profile of a portfolio, which is fundamental to take optimal decisions on the capital allocation (see e.g. Glasserman \& $\mathrm{Xu}, 2014)$. Among the methods for assessing financial risk, Gaussian distribution-based procedures

\footnotetext{
${ }^{*}$ Corresponding author.
} 
are undoubtedly of large popularity (Duffie \& Pan, 1997; Chang et al., 2015). However, since asset returns are usually fat-tailed (see e.g. Viswanathan et al., 2003; Ausloos \& Ivanova, 2003; Krause, 2006; Zhou, 2009; Filiasi et al., 2014; Dhesi \& Ausloos, 2016; Caginalp \& Caginalp, 2018), the use of Gaussian processes leads to an underestimation of the risk (Rachev et al., 2005; Kim et al., 2012). In this context, we mention Value-at-risk (VaR), introduced by JP Morgan (see Longerstaey \& Zangari, 1996), which represents a standard measure in financial risk management and exhibits some relevant positive aspects such as simplicity, computational facility and quick applicability. However, it has been deeply proved and discussed that VaR has several conceptual problems and presents some weaknesses (Frey \& McNeil, 2002): it is an unstable and numerically difficult to use method when the losses do not follow a Gaussian distribution (Ferraty \& Quintela-Del-Río, 2016), which is usually the case in the analysis of financial data (see e.g. Pafka \& Kondor, 2001; Caporin, 2008). For this reason, other approaches to VaR have been employed. Such approaches extend VaR in two directions: first, they consider non-Gaussian distributions of returns (Giot \& Laurent, 2003; Chen et al., 2012; Zhao et al., 2015); second, they overcome the drawbacks of the constitutive elements of the Value-atRisk. Indeed, such a risk measure does not distinguish between slightly higher losses and potentially fatal losses (Krzemienowski \& Szymczyk, 2016), being sometimes too optimistic in its estimates: VaR measures only percentiles of profit-loss distributions, and thus disregards any loss beyond the VaR level, or "tail risk" (Berkowitz \& O'Brien, 2002). Moreover, VaR is not always sub-additive (Stoyanov et al., 2013), meaning that it is not always able to identify diversification opportunities. For all these reasons, literature has proposed the Conditional VaR (CVaR, see Rockafellar \& Uryasev, 2001; Acerbi \& Tasche, 2002; Huang et al., 2010) as an alternative to VaR, which must be seen as complementary to its predecessor (Alexander \& Baptista, 2004; Yamai \& Yoshiba, 2005). Indeed, the CVaR estimates are affected by whether large and infrequent loss is realized in the obtained sample, since expected shortfall considers the right tail of the loss distribution. Therefore, when the underlying loss distribution becomes more fat-tailed, the CVaR estimates become more varied due to infrequent and large losses, and their estimation error grows larger than VaR's. Futhermore, CVaR is a coherent risk measure (Artzner et al., 1999; Inui \& Kijima, 2005) and, differently from $\mathrm{VaR}$, it models the very important empirical evidence stating that diversification reduces risk. The arguments above explain the popularity of $\mathrm{CVaR}$ as risk measure in the context of portfolio theory (see e.g. Krokhmal et al., 2002; Ciliberti \& Mézard, 2007; Zhang \& Liu, 2017).

Beyond the distributions of the returns, a portfolio risk assessment procedure also has to consider the dependence structure among the assets. In this respect, one of the most authoritative proposals is the employment of copula functions (see e.g. Nelsen, 2006). Under a mathematical perspective, copulas are special functions with a strong connection with multivariate probability laws. Such a connection is grounded on the ability of a copula to describe the stochastic dependence among a set of random variables. Specifically, a copula function can be used to transform the marginal 
distributions of a set to random variables in their joint distribution, hence inferring their stochastic structure (Sklar's Theorem, 1959). In the present study, we take copulas for modeling the stochastic dependence among the returns, in accord to a wide number of literature contribution (see e.g. Chen et al., 2014; Zhang et al., 2014; Domino \& Blachowicz, 2015; Gonzalez-Pedraz et al., 2015; Choe et al., 2015; Han et al., 2016; Cerqueti et al., 2018; Yu et al., 2018). Thus, the problem can be split into two separate parts: first, to identify the marginal distributions of the returns of the single assets; second, to identify the specific copula which is more appropriate for representing the dependence structure of the returns, according to Sklar (1959).

This paper deals with this important topic. We introduce the family of the Generalized Error Distribution (G.E.D.) with shape parameter $p$ for capturing the distribution of the returns and, accordingly, provide an estimation of $p$ through the $L p_{\min }$ method (Giacalone, 1997; Giacalone \& Richiusa, 2006). For a discussion of the superiority of the $L p_{\min }$ method for the estimation procedure, refer to the Appendix. The G.E.D. is particularly suitable for our purpose. Indeed, it has been used quite extensively in univariate time series with financial applications (Koutmos, 1998; So et al., 2008) and has demonstrated an excellent flexibility in the estimation process, as it embeds other well-known distributions including the Gaussian one (He \& Gong, 2009). Moreover, we propose a bivariate setting and a copula-based method for modeling the stochastic dependence among the assets. In particular, we refer to two cases: the Gaussian copula, which is the considered extended copula and serves here as benchmark (see e.g. Malavergne \& Sornette, 2003; Demarta \& McNeil, 2005); a modified Gaussian copula, where the correlation coefficient of the Gaussian case is replaced by a generalization of it obtained as the correlation parameter of a bivariate G.E.D. (Taguchi, 1974; Sultan, 2007). In such two contexts we propose the computation of the CVaR for assessing the risk of a generic portfolio created by the considered assets.

Our approach is empirically validated by the computation of the CVaR of a large set of portfolios generated by General Motors Company and Ford Motor Company, with daily quotations ranging in the period 2012-2016. Results confirm the superiority of the G.E.D. with respect to the Gaussian copula method in terms of assessing the risk in a more prudential way.

The rest of the paper is organized as follows. In Section 2, we introduce the quantitative ingredients of the study, with the main definitions. The proposed methodological setting for calculating the Conditional Value-at-Risk of a generic portfolio is presented in Section 3. Section 4 presents an empirical application, on which the capacity of the new method of providing an estimation of the risk is shown. In Section 5 some conclusive remarks are given. The Appendix contains a discussion of the validity of the $L p_{\min }$ method as a device for the estimation of $p$. 


\section{Main definitions and preliminaries}

This section contains a description of the methodological instruments which will be used throughout the paper.

\subsection{The Generalized Error Distribution}

The Generalized Error Distribution (G.E.D.) family was introduced by Subbotin (1923) and has been employed by various authors with different names and parameterizations (e.g. Box \& Tiao, 1973; Agrò, 1999; Mineo, 2007; Bottazzi \& Secchi, 2011). A parameterization of the G.E.D. density function for a random variable $X$ is:

$$
f\left(x ; \mu, \sigma_{p}, p\right)=\frac{1}{2 \sigma_{p} p^{1 /(p)} \Gamma(1+1 / p)} \exp \left(-\frac{1}{p}\left|\frac{x-\mu}{\sigma_{p}}\right|^{p}\right) \quad \text { for }-\infty<\mathrm{x}<\infty
$$

where $\mu=E(X)$ is the location parameter, $\sigma_{p}=\left[E|X-\mu|^{p}\right]^{1 / p}>0$ is the scale parameter, $p>0$ is the shape parameter and $\Gamma$ is the Euler Gamma function.

The density of a generic G.E.D. distribution is unimodal, symmetric and, for $p>1$, bell-shaped. As particular cases we obtain the Laplace distribution $(p=1)$, the Normal $(p=2)$ and the Uniform $(p \rightarrow \infty)$; for values of $1<p<2$ we obtain leptokurtic densities which are more fat-tailed than the Normal and for values of $p>2$ we obtain platykurtic densities (see Fig. 1).

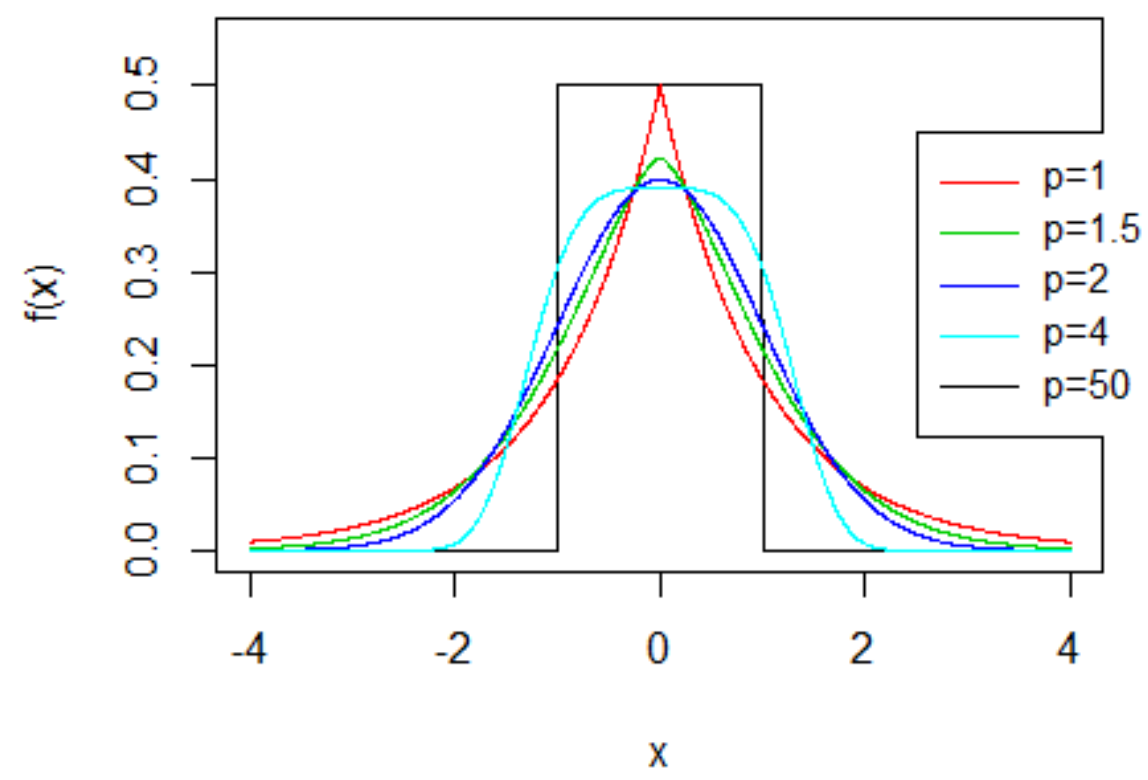

Figure 1: Graphical representation of the Generalized Error Distribution density function as the value of the parameter $p$ varies. The cases of $p=1,1.5,2,4,50$ have been taken into account.

Thus, the G.E.D. represents a generalization of a large set of distributions. Therefore, assuming a G.E.D. for financial data allows for a better description of them and leads to good estimates of 
the maximum losses to be expected.

When analyzing real data using the G.E.D., a fundamental problem is to obtain a good estimation of the $p$ parameter when it is unknown. To estimate it, it is possible to use methods based on kurtosis indexes or on maximum likelihood functions.

In the estimation of $p$, a relevant role is played by the ratio $\beta_{k}$ between the moments of order $2 k$ and the squared moment of order $k$ (Giacalone \& Richiusa, 2006). Such a ratio depends on the $p$ shape parameter, and is also called "Generalized Kurtosis" (Mineo, 1989):

$$
\beta_{k}=\frac{\mu_{2 k}}{\mu_{k}^{2}}=\frac{\Gamma(1 / p) \Gamma((2 k+1) /(p))}{[\Gamma((k+1) /(p))]^{2}},
$$

where $\mu_{k}$ is the moment of order $k$.

If $k=2$, then the Generalized Kurtosis is usually denoted as "Pearson kurtosis".

By considering the square root of the reciprocal of $\beta_{k}$ in $(2)$ for the case $k=1$, we obtain the "Geary length-of-tails index", introduced in Geary (1936) and defined as follows:

$$
I=\frac{\mu_{1}}{\sqrt{\mu_{2}}}=\frac{\Gamma(2 / p)}{\sqrt{\Gamma(1 / p) \Gamma(3 / p)}} .
$$

The indexes $I$ and $\beta_{2}$ show a different behavior according to the variation of $p$ (see e.g. Giacalone, 1997). We present some values of $I$ and $\beta_{2}$ as $p$ varies, to give a clear idea about the distribution we deal with and its versatility (see Table 1). As we see, the G.E.D. allows for a great range of distributions with different bell forms and different thickness of tails.

Calculating the sample values of $I$ and $\beta_{2}$, it is possible to obtain, by inverse interpolation, two different estimations of $p$.

Kendall \& Stuart (1966) and Gonin \& Money (1987) considered the unbiased estimates of the second and fourth order sample moments with correction factors depending on the sample size $n$. The authors denoted such corrected moments as $\hat{\mu_{2}}$ and $\hat{\mu_{4}}$, respectively, and achieved a corrected estimation of $\beta_{2}$ and $I$ as

$$
\hat{\beta_{2}}=\frac{\hat{\mu_{4}}}{{\hat{\mu_{2}}}^{2}}, \quad \hat{I}=\frac{\hat{\mu_{1}}}{\sqrt{\hat{\mu_{2}}}} .
$$

On this ground, it is possible to find out in the literature several proposals to estimate the $p$ shape parameter (see e.g. Harter, 1977; Money et al., 1982; Sposito, 1982; Mineo, 1989; Agrò, 1995; Giacalone, 1997; Agrò, 1999; Mineo \& Ruggieri, 2005; Mineo, 2007).

\subsection{The Generalized Correlation Coefficient and the G.E.D. Copula}

A bivariate copula is a function $C:[0,1]^{2} \rightarrow[0,1]$ whose main interest in the field of probability is that it associates univariate marginal distributions to their joint ones (Sklar, 1959). Formally, if $F$ is a bivariate distribution function of marginals $F_{1}$ and $F_{2}$, then there exists a bivariate copula $C$ such that:

$$
F\left(x_{1}, x_{2}\right)=C\left(F_{1}\left(x_{1}\right), F_{2}\left(x_{2}\right)\right), \quad\left(x_{1}, x_{2}\right) \in \mathbf{R}^{2} .
$$




\begin{tabular}{|c|c|c|c|c|c|}
\hline$p$ & $\beta_{2}$ & $I$ & $p$ & $\beta_{2}$ & $I$ \\
\hline 0.5 & 25.20000 & .54772 & 3.0 & 2.41840 & .82732 \\
\hline 0.8 & 8.56514 & .66392 & 3.2 & 2.35716 & .83082 \\
\hline 1.0 & 6.00000 & .70711 & 3.4 & 2.30827 & .83993 \\
\hline 1.2 & 4.74348 & .73696 & 3.6 & 2.26064 & .83651 \\
\hline 1.4 & 4.01786 & .75891 & 3.8 & 2.22208 & .83879 \\
\hline 1.6 & 3.55270 & .77503 & 4.0 & 2.18844 & .84090 \\
\hline 1.8 & 3.23236 & .78776 & 4.2 & 2.15889 & .84273 \\
\hline 2.0 & 3.00000 & .79788 & 4.4 & 2.13276 & .84436 \\
\hline 2.2 & 2.82473 & .80609 & 4.6 & 2.10952 & .84583 \\
\hline 2.4 & 2.68841 & .81285 & 4.8 & 2.08875 & .84715 \\
\hline 2.6 & 2.57977 & .81849 & 5.0 & 2.07010 & .84834 \\
\hline 2.8 & 2.49143 & .82326 & 10.0 & 1.88416 & .86054 \\
\hline
\end{tabular}

Table 1: Pearson kurtosis and Geary length-of-tails values in function of $\mathrm{p}$

If the marginal distribution functions are continuous, then the copula function satisfying (5) is unique.

Formula (5) highlights the role of copulas in expressing the dependence between $n$ stochastic variables. For a detailed view of the concept of copula and of its properties, refer to Nelsen (2006), Jaworski et al. (2010), McNeil et al. (2015).

There are various copula functions in the literature (see McNeil et al., 2015) and others can be introduced, with full respect for the formal properties (Nelsen, 2006), in order to capture the different dependence structures among stochastic variables. We are here interested in the bivariate Gaussian 
copula, which is defined as:

$$
C(u, v \mid \rho)=\int_{-\infty}^{\Phi^{-1}(u)} \int_{-\infty}^{\Phi^{-1}(v)} \frac{1}{2 \pi \sqrt{\left(1-\rho^{2}\right)}} \exp \left\{\frac{-\left(r^{2}-2 \rho r s+s^{2}\right)}{2\left(1-\rho^{2}\right)}\right\} d r d s
$$

where $\Phi^{-1}$ is the inverse of Gaussian distribution function, $(u, v)$ uniform independent random variables generated from $(\mathrm{X}, \mathrm{Y})$ random variables, and $\rho \in[-1,1]$ is a parameter representing the Pearson's correlation coefficient associated to the bivariate normal.

The G.E.D. copula for a generic random vector $(X, Y)$ is obtained by transforming the Gaussian copula. Specifically, the parameter $\rho$ is replaced by the Generalized Correlation Coefficient $\rho_{p} \in$ $[-1,1]$, introduced by Taguchi (1974) as the correlation parameter of a bivariate Generalized Error Distribution, and defined as in Sultan (2007). See the next Section for an explicit estimation of $\rho_{p}$.

\section{The methodology}

We start from two sets of consecutive observations of the returns of two assets:

$$
X=\left\{x_{1}, \ldots, x_{n}\right\}, \quad Y=\left\{y_{1}, \ldots, y_{n}\right\}
$$

We assume that $X$ and $Y$ are empirically distributed according to a G.E.D., and refer hereafter to $X$ and $Y$ as random variables when needed.

For what concerns the stochastic dependence between $X$ and $Y$, we propose two different frameworks: in the benchmark case, such a dependence is described by a Gaussian copula; in the other case, it is described by a G.E.D. copula.

According to (5), we are able to derive the joint distribution function of the random vector $(X, Y)$ in the two cases of copula. Such a joint distribution is needed for computing the VaR and the CVaR at a confidence level $c$ for a generic portfolio with return $P=\alpha X+\beta Y$, where $\alpha, \beta \in \mathbf{R}$ such that $\alpha+\beta=1$.

The parameter $\rho_{p}$ of the G.E.D. copula is estimated as follows:

$$
\rho_{p}=\frac{\operatorname{codisp}{ }^{(p)}(X, Y)}{\sigma_{p}(X) \sigma_{p}(Y)}
$$

where

$$
\begin{gathered}
\left|\operatorname{codisp}^{(p)}(X, Y)\right|^{p}=\left|E\left[\left(Y-\mu_{Y}\right)\left|X-\mu_{X}\right|^{p-1} \operatorname{sign}\left(X-\mu_{X}\right)\right]\right| \cdot\left|E\left[\left(X-\mu_{X}\right)\left|Y-\mu_{Y}\right|^{p-1} \operatorname{sign}\left(Y-\mu_{Y}\right)\right]\right|, \\
\sigma_{p}(X)=\left[E\left|X-\mu_{X}\right|^{p}\right]^{1 / p}, \quad \sigma_{p}(Y)=\left[E\left|Y-\mu_{Y}\right|^{p}\right]^{1 / p}
\end{gathered}
$$

and $\mu_{X}$ and $\mu_{Y}$ are the expected values of $X$ and $Y$ as statistical variables, respectively. By definition, we have $-1 \leq \rho_{p} \leq 1$.

Indeed, the G.E.D. provides a generalization of every $p$-norm statistical index, valid for each value 
of $p$. In particular, the codisp index used above, for instance, is a generalization of covariance, since $\operatorname{codisp}^{(p)}(X, Y)$ is the covariance between $X$ and $Y$ when $p=2$.

The parameters $\mu, p$ and $\sigma_{p}$ are estimated using the $L p_{\min }$ method (Giacalone, 1997; Giacalone \& Richiusa, 2006), based on the definitions of $I$ and $\beta_{2}$ in (2) and (3). For a description of the estimation procedure and a comparison with other approaches employed in the literature, see the Appendix.

The fundamental steps in the algorithm for the computation of the CVaR of a portfolio $(\alpha, \beta)$, for both cases of Gaussian and G.E.D. copulas, are:

1. estimation of the parameters $\mu, p, \sigma_{p}$ in (1) for the two series of returns $X$ and $Y$. In accord to the notation used above, we will denote the parameters as $\mu_{X}, p_{X}, \sigma_{p, X}$ and $\mu_{Y}, p_{Y}, \sigma_{p, Y}$;

2. estimation of the $\rho_{p}$ parameter of the G.E.D. copula by using formula (7), with $p=\alpha p_{X}+\beta p_{Y}$;

3.1 generation of couples $(x, y)$, which are the realization of the double stochastic variable $(X, Y)$ having G.E.D. marginals identified by the parameters found in item 1. and stochastic dependence described by the G.E.D. copula with $\rho_{p}$ of item $2 . ;$

3.2 generation of couples $(x, y)$, which are the realization of the double stochastic variable $(X, Y)$ having G.E.D. marginals of item 1. and stochastic dependence described by the Gaussian copula;

4. construction of the realizations of the returns of portfolio $P=\alpha X+\beta Y$ and of its empirical distribution, in both of cases of items 3.1 and 3.2;

5. computation of the Value-at-Risk of $P$ at a confidence level $(1-c)$, in both of cases of items 3.1 and 3.2. As we will see in the empirical experiments, $c$ will be set to $5 \%$, according to the standard applications of CVaR;

6. computation of the Conditional Value-at-Risk of $P$ at a confidence level $(1-c) \%$, in both of cases of items 3.1 and 3.2 .

As we will see in the next Section, the algorithm above is repeated for all the considered portfolios, given by $\alpha=0.01: 0.01: 0.99$ and $\beta=1-\alpha$.

\section{Application and results}

In order to evaluate and compare the performances of the CVaRs obtained for the two copulas, we consider two time series of equities, traded on the New York Stock Exchange ${ }^{1}$ :

\footnotetext{
${ }^{1}$ All the necessary calculations have been implemented and processed on the statistical environment R.
} 
1. General Motors Company, first Vehicle Manufacturing Group by volume in the United States of America.

2. Ford Motor Company, second Vehicle Manufacturing Group by volume in the United States of America.

The data used refer to the daily quotations for all the trading days in the years 2012-2016, for a total of 1258 data (data source: Investing.com).

Each time series of daily prices has been transformed into a series of daily logarithmic returns.

The level of confidence for the computation of the CVaR has been set at $1-c=95 \%$. We denote by $X$ and $Y$ the obtained samples of returns for General Motors Company and Ford Motor Company, respectively.

The estimates of the $p$ shape parameters for the two series are $\hat{p}_{X}=1.283$ for General Motors and $\hat{p}_{Y}=1.243$ for Ford. Hence, the distributions of the returns are leptokurtic and more fat-tailed than the Gaussian one. Figures 2 and 3 show the returns of the two single assets, with the adaptation of a Gaussian distribution and the estimated Generalized Error Distribution.

We then generated 99 portfolios $(\alpha, \beta)$, with $\alpha=0.01: 0.01: 0.99$ and $\beta=1-\alpha$, with return $P=\alpha X+\beta Y$.

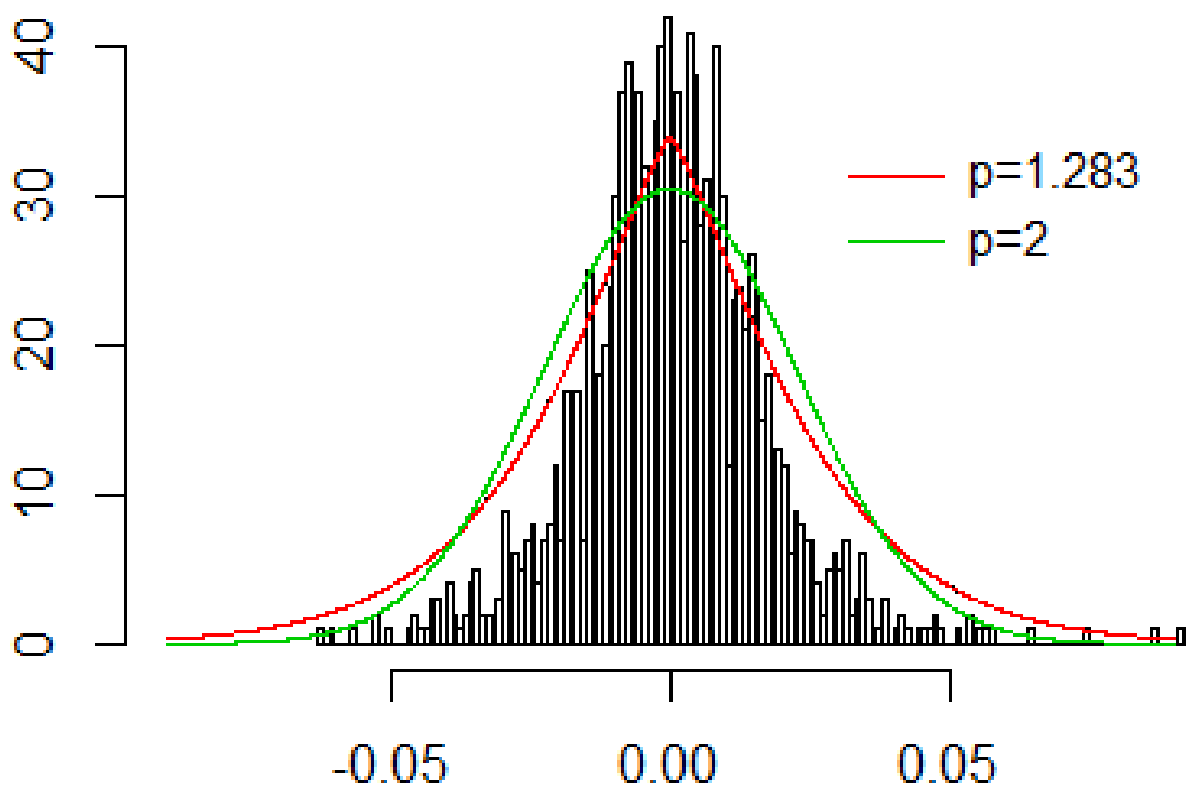

Figure 2: Case of General Motors Company dataset. Histogram of the returns with juxtaposition of G.E.D. density function, whose calibrated parameter is $\hat{p}=1.283$. The G.E.D. density function with $p=2$ is also inserted, for comparison purposes.

The CVaR has been computed in both of cases of Gaussian and G.E.D. copulas. First, CVaR has been calculated for the two assets, revealing that in the Gaussian case it is -0.0340 for General 


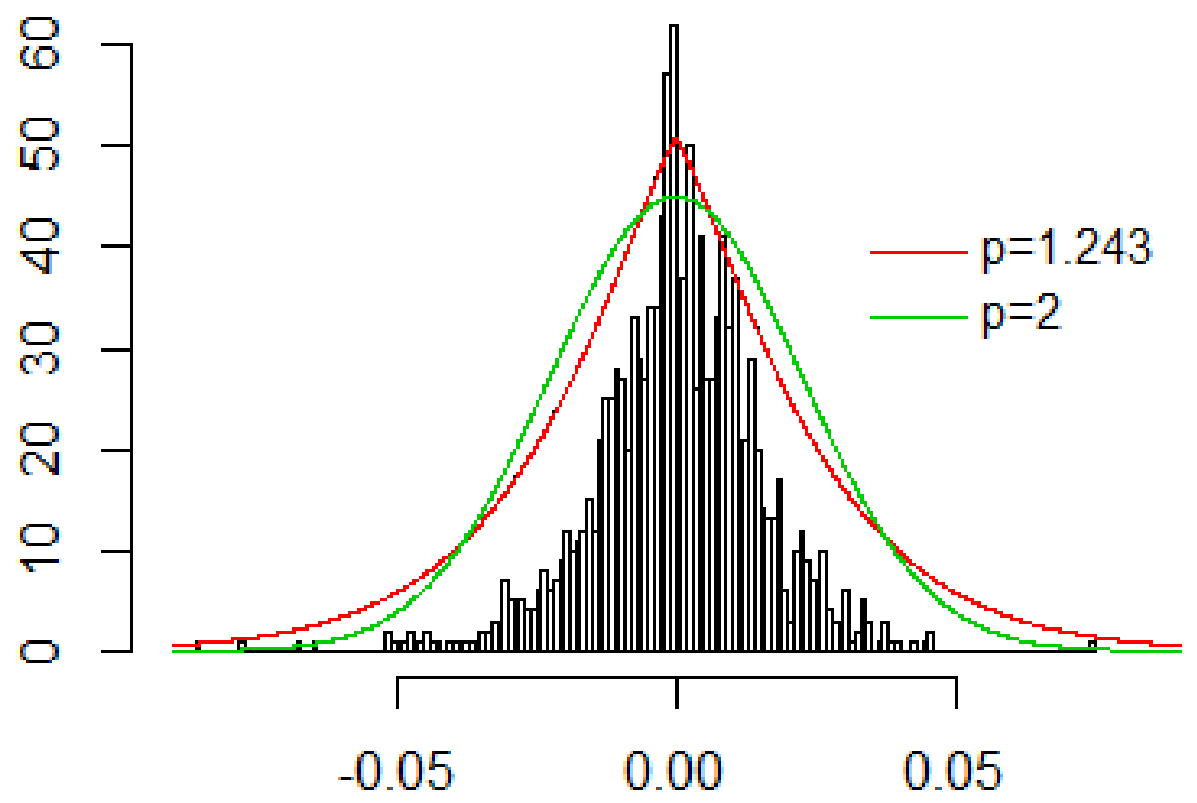

Figure 3: Case of Ford Motor Company dataset. Histogram of the returns with juxtaposition of G.E.D. density function, whose calibrated parameter is $\hat{p}=1.243$. The G.E.D. density function with $p=2$ is also inserted, for comparison purposes.

Motors and -0.0309 for Ford, while in the G.E.D. case it is -0.0330 for General Motors and -0.0420 for Ford.

While there is a small difference in the two General Motors CVaRs, Ford data reveal a significantly bigger CVaR when analyzed through the G.E.D. method.

Then, we calculated the CVaR for the 99 portfolios in both of cases of Gaussian and G.E.D. copulas (see Figure 5). For a view of the values of the portfolios' $p$ parameters, see Figure 4.

From Figure 5, we see that the CVaR is smaller in the case of Gaussian copula than in the one of G.E.D. copula in 86 cases out of 99. This gives that G.E.D. is broadly more prudential than Gaussian in estimating risk, with the exception of the corner cases of $\alpha$ 's close to one. This outcome suggests that a risk-averse individual should generally prefer a G.E.D. copula rather than a Gaussian one when considering the stochastic dependence among her/his portfolio assets.

\section{Conclusions}

The present paper is part of market risk calculation methods, whose purpose is to support risk managers' decision-making processes. Among the different methods proposed in the literature for calculating Value-at-Risk, we took into account the well-known RiskMetrics. After examining the most useful methods that consider the CVaR approach, we proposed the G.E.D. method and eval- 


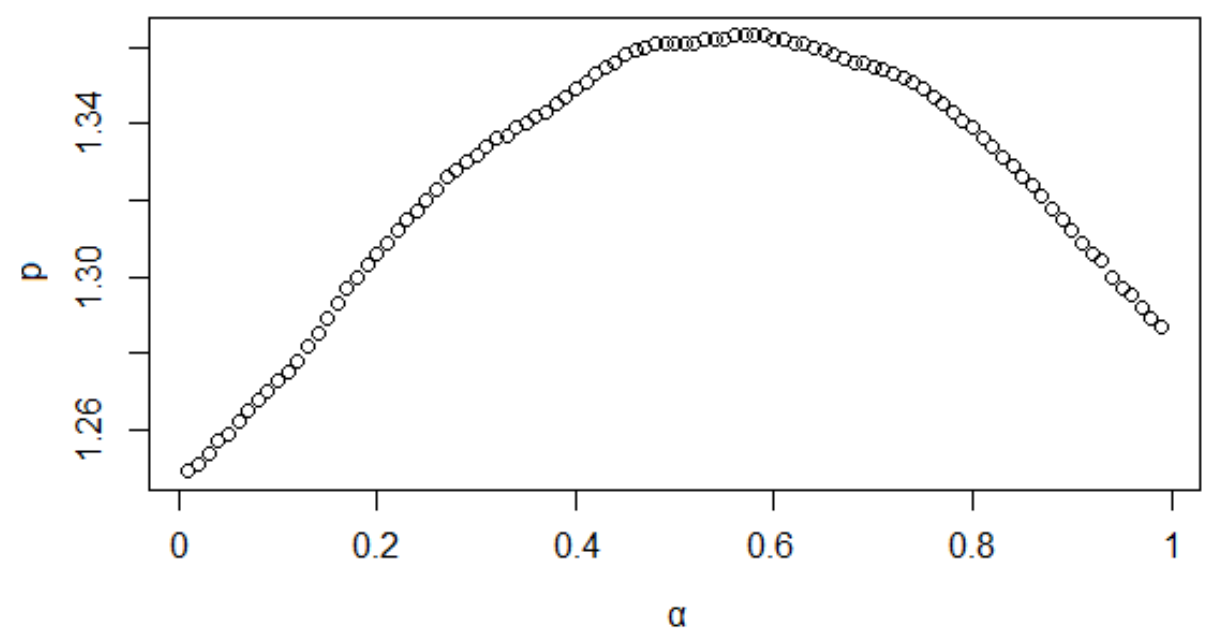

Figure 4: Scatter plot of the values of the $p$ shape parameter for the 99 considered portfolios.

uated its performance compared to the RiskMetrics one.

We introduced the new G.E.D. method and its properties. For the first time, the G.E.D. copula was proposed as a generalization of the Gaussian copula, which is just a special case of the first one, for the $p=2$ case. Moreover, we introduced the Generalized Correlation Coefficient of norm $p$ that, for the $p=2$ case, equals the classic Bravais-Pearson correlation coefficient. We then presented an algorithm, tested on simulated returns, with the aim of verifying the performance of the new method over the classical ones. The results obtained confirm the higher performance of the G.E.D. method, while the assumption of normality of the returns distribution determines confidence intervals with the lowest predictive power. The assumption of normality, subject to verification, was rejected as the returns of all the examined stocks have fat-tailed distribution and kurtosis characteristics which are neglected by RiskMetrics. It does seem that CVaR computed in the G.E.D. case can constitute a valid generalization of CVaR computed under the RiskMetrics approach, mainly when the distributions are not fat-tailed.

\section{References}

[1] Acerbi, C., \& Tasche, D. (2002). On the coherence of expected shortfall. Journal of Banking \& Finance, 26(7), 1487-1503.

[2] Agrò, G. (1995). Maximum likelihood estimation for the Exponential Power Function Parameters. Communications in Statistics - Simulation and Computation, 24(2), 523-536.

[3] Agrò, G. (1999). Parameter orthogonality and Conditional Profile Likelihood: the Exponential Power Function Case. Communications in Statistics - Theory and Methods, 28(8), 1759-1768. 


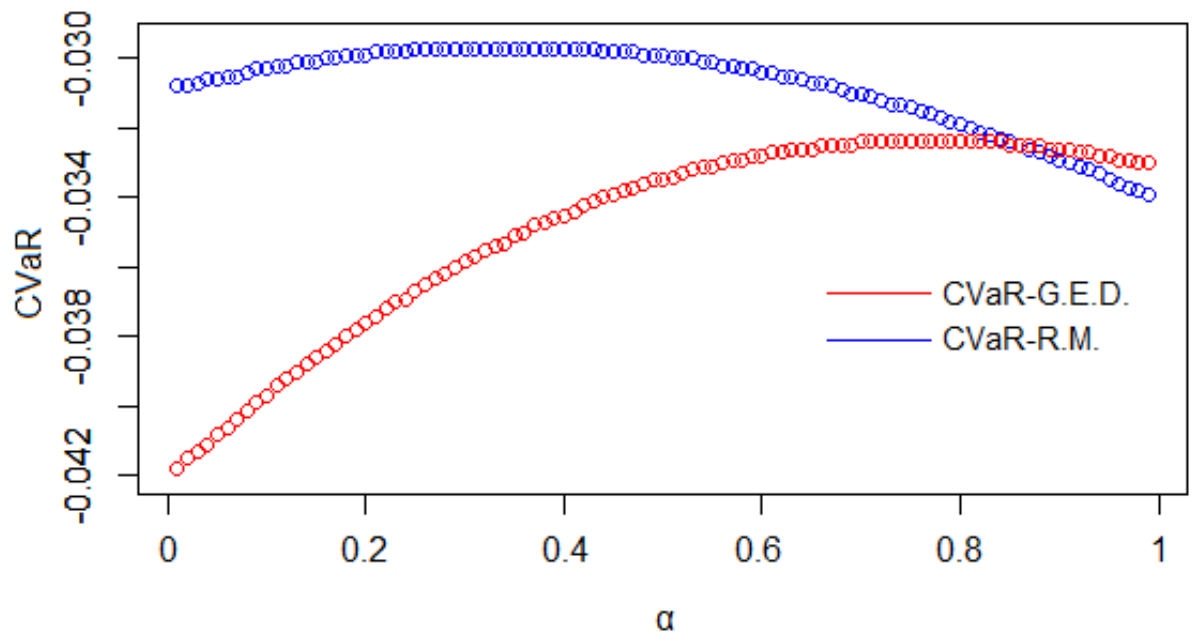

Figure 5: Values of the CVaR for the 99 portfolios computed in both cases of Gaussian copula used in RiskMetrics (CVaR-R.M., in the legend) and G.E.D. copula (CVaR-G.E.D., in the legend).

[4] Alexander, G. J., \& Baptista, A. M. (2004). A comparison of VaR and CVaR constraints on portfolio selection with the mean-variance model. Management Science, 50(9), 1261-1273.

[5] Artzner, P., Delbaen, F., Eber, J. M., \& Heath, D. (1999). Coherent measures of risk. Mathematical Finance, 9(3), 203-228.

[6] Ausloos, M., \& Ivanova, K. (2003). Dynamical model and nonextensive statistical mechanics of a market index on large time windows. Physical Review E, 68(4), 046122.

[7] Berkowitz, J., \& O'Brien, J. (2002). How accurate are value-at-risk models at commercial banks?. The Journal of Finance, 57(3), 1093-1111.

[8] Bottazzi, G., \& Secchi, A. (2011). A new class of asymmetric exponential power densities with applications to economics and finance. Industrial and Corporate Change, 20(4), 991-1030.

[9] Box, G. E., \& Tiao, G. C. (1973). Bayesian inference in statistical analysis. Addison-Wesley.

[10] Caginalp, C., \& Caginalp, G. (2018). The quotient of normal random variables and application to asset price fat tails. Physica A: Statistical Mechanics and its Applications, 499, 457-471.

[11] Caporin, M. (2008). Evaluating value-at-risk measures in the presence of long memory conditional volatility. The Journal of Risk, 10(3), 79-110.

[12] Cerqueti, R., Rotundo, G., \& Ausloos, M. (2018). Investigating the Configurations in CrossShareholding: A Joint Copula-Entropy Approach. Entropy, 20(2), 134. 
[13] Chang, C. L., Jiménez-Martín, J. Á., Maasoumi, E., \& Pérez-Amaral, T. (2015). A stochastic dominance approach to financial risk management strategies. Journal of Econometrics, 187(2), $472-485$.

[14] Chen, Q., Gerlach, R., \& Lu, Z. (2012). Bayesian Value-at-Risk and expected shortfall forecasting via the asymmetric Laplace distribution. Computational Statistics \& Data Analysis, 56(11), 3498-3516.

[15] Chen, W., Wei, Y., Lang, Q., Lin, Y., \& Liu, M. (2014). Financial market volatility and contagion effect: A copulamultifractal volatility approach. Physica A: Statistical Mechanics and its Applications, 398, 289-300.

[16] Choe, G. H., Jang, H. J., \& Kwon, S. W. (2015). A factor contagion model for portfolio credit derivatives. Quantitative Finance, 15(9), 1571-1582.

[17] Ciliberti, S., \& Mézard, M. (2007). Risk minimization through portfolio replication. The European Physical Journal B, 57(2), 175-180.

[18] Demarta, S., \& McNeil, A. J. (2005). The t copula and related copulas. International statistical review, 73(1), 111-129.

[19] Dhesi, G., \& Ausloos, M. (2016). Modelling and measuring the irrational behaviour of agents in financial markets: Discovering the psychological soliton. Chaos, Solitons and Fractals, 88, 119-125.

[20] Domino, K., \& Blachowicz, T. (2015). The use of copula functions for modeling the risk of investment in shares traded on world stock exchanges. Physica A: Statistical Mechanics and its Applications, 424, 142-151.

[21] Duffie, D., \& Pan, J. (1997). An overview of value at risk. The Journal of Derivatives, 4(3), $7-49$.

[22] Ferraty, F., \& Quintela-Del-Río, A. (2016). Conditional VaR and expected shortfall: a new functional approach. Econometric Reviews, 35(2), 263-292.

[23] Filiasi, M., Livan, G., Marsili, M., Peressi, M., Vesselli, E., \& Zarinelli, E. (2014). On the concentration of large deviations for fat tailed distributions, with application to financial data. Journal of Statistical Mechanics: Theory and Experiment, 2014(9), P09030.

[24] Frey, R., \& McNeil, A. J. (2002). VaR and expected shortfall in portfolios of dependent credit risks: conceptual and practical insights. Journal of Banking \& Finance, 26(7), 1317-1334.

[25] Geary, R.C. (1936). The Distribution of the "Student's" Ratio for the Non-Normal Samples. Supplement to the Journal of the Royal Statistical Society, 3(2), 178-184. 
[26] Giacalone, M. (1997). Lp-norm estimators for nonlinear regression models. Student, 2, 119-130.

[27] Giacalone, M., \& Richiusa, R. (2006). Lp-norm estimation: some simulation studies in presence of multicollinearity. Student, 5, 235-246.

[28] Giot, P., \& Laurent, S. (2003). Market risk in commodity markets: a VaR approach. Energy Economics, 25(5), 435-457.

[29] Glasserman, P., \& Xu, X. (2014). Robust risk measurement and model risk. Quantitative Finance, 14(1), 29-58.

[30] Gonin, R., \& Money, A. H. (1987). A review of computational methods for solving the nonlinear L1 norm estimation problem. Statistical data analysis based on the L1 norm and related methods, Ed. Y. Dodge, North Holland.

[31] Gonzalez-Pedraz, C., Moreno, M., \& Pena, J. I. (2015). Portfolio selection with commodities under conditional copulas and skew preferences. Quantitative Finance, 15(1), 151-170.

[32] Han, Y., Gong, P., \& Zhou, X. (2016). Correlations and risk contagion between mixed assets and mixed-asset portfolio VaR measurements in a dynamic view: An application based on time varying copula models. Physica A: Statistical Mechanics and its Applications, 444, 940-953.

[33] Harter, H. (1977). Nonuniqueness of least absolute values regression. Communications in Statistics - Theory and Methods, 6(9), 829-838.

[34] He, X., \& Gong, P. (2009). Measuring the coupled risks: A copula-based CVaR model. Journal of Computational and Applied Mathematics, 223(2), 1066-1080.

[35] Huang, D., Zhu, S., Fabozzi, F. J., \& Fukushima, M. (2010). Portfolio selection under distributional uncertainty: A relative robust CVaR approach. European Journal of Operational Research, 203(1), 185-194.

[36] Inui, K., \& Kijima, M. (2005). On the significance of expected shortfall as a coherent risk measure. Journal of Banking \& Finance, 29(4), 853-864.

[37] Jaworski, P., Durante, F., Hardle, W. K., \& Rychlik, T. (2010). Copula theory and its applications. Springer.

[38] Kendall, M. C., \& Stuart, A. (1966). The Advanced Theory of Statistics. Griffin, London.

[39] Kim, Y. S., Giacometti, R., Rachev, S. T., Fabozzi, F. J., \& Mignacca, D. (2012). Measuring financial risk and portfolio optimization with a non-Gaussian multivariate model. Annals of Operations Research, 201(1), 325-343. 
[40] Koutmos, G. (1998). Asymmetries in the conditional mean and the conditional variance: Evidence from nine stock markets. Journal of Economics and Business, 50(3), 277-290.

[41] Krause, A. (2006). Fat tails and multi-scaling in a simple model of limit order markets. Physica A: Statistical Mechanics and its Applications, 368(1), 183-190.

[42] Krokhmal, P., Palmquist, J., \& Uryasev, S. (2002). Portfolio optimization with conditional value-at-risk objective and constraints. Journal of Risk, 4, 43-68.

[43] Krzemienowski, A., \& Szymczyk, S. (2016). Portfolio optimization with a copula-based extension of conditional value-at-risk. Annals of Operations Research, 237(1-2), 219-236.

[44] Longerstaey, J., \& Zangari, P. (1996). RiskMetrics Technical Document. J.P. Morgan, Fourth Edition, New York.

[45] Malevergne, Y., \& Sornette, D. (2003). Testing the Gaussian Copula hypothesis for financial assets dependences. Quantitative Finance, 3, 231-250.

[46] McNeil, A. J., Frey, R., \& Embrechts, P. (2015). Quantitative risk management: Concepts, techniques and tools. Princeton University Press.

[47] Mineo, A. (1989). The norm-p estimation of location, scale and simple linear regression parameters. In Statistical Modelling, 222-233, Springer.

[48] Mineo, A. M., \& Ruggieri, M. (2005). A software tool for the exponential power distribution: The normalp package. Journal of Statistical Software, 12(4), 1-24.

[49] Mineo, A. M. (2007). On the estimation of the structure parameter of a normal distribution of order p. Statistica, 63(1), 109-122.

[50] Money, A. H., Affleck-Graves, J. F., Hart, M. L., \& Barr, G. D. I. (1982). The linear regression model: Lp norm estimation and the choice of p. Communications in Statistics - Simulation and Computation, 11(1), 89-109.

[51] Nelsen, R. B. (2006). An Introduction to Copulas, 2nd edition. Springer, New York.

[52] Pafka, S., \& Kondor, I. (2001). Evaluating the RiskMetrics methodology in measuring volatility and Value-at-Risk in financial markets. Physica A: Statistical Mechanics and its Applications, 299(1), 305-310.

[53] Rachev, S. T., Menn, C., \& Fabozzi, F. J.(2005). Fat-tailed and skewed asset return distributions: implications for risk management, portfolio selection, and option pricing. John Wiley \& Sons. 
[54] Rockafellar, R. T., \& Uryasev, S. (2001). Conditional value-at-risk for general loss distributions. Journal of Banking \& Finance, 26(7), 1443-1471.

[55] Sklar, M. (1959). Fonctions de répartition à n dimensions et leurs marges. Université Paris 8.

[56] So, M. K., Chen, C. W., Lee, J. Y., \& Chang, Y. P. (2008). An empirical evaluation of fat-tailed distributions in modeling financial time series. Mathematics and Computers in Simulation, $77(1), 96-108$.

[57] Sposito, V. A. (1982). On unbiased Lp regression estimators. Journal of the American Statistical Association, 77(379), 652-653.

[58] Stoyanov, S. V., Rachev, S. T., \& Fabozzi, F. J. (2013). Sensitivity of portfolio VaR and CVaR to portfolio return characteristics. Annals of Operations Research, 205(1), 169-187.

[59] Subbotin, M. (1923). On the Law of Frequency of Error. Mathematicheskii Sbornik.

[60] Sultan, K. S. (2007). Order statistics from the generalized exponential distribution and applications. Communications in Statistics - Theory and Methods, 36(7), 1409-1418.

[61] Taguchi, T. (1974). On Fechner's thesis and statistics with Norm-p. Annals of the Institute of Statistical Mathematics, 26(1), 175-193.

[62] Viswanathan, G. M., Fulco, U. L., Lyra, M. L., \& Serva, M. (2003). The origin of fat-tailed distributions in financial time series. Physica A: Statistical Mechanics and its Applications, $329(1-2), 273-280$.

[63] Yamai, Y., \& Yoshiba, T. (2005). Value-at-risk versus expected shortfall: A practical perspective. Journal of Banking \& Finance, 29(4), 997-1015.

[64] Yu, W., Yang, K., Wei, Y., \& Lei, L. (2018). Measuring Value-at-Risk and Expected Shortfall of crude oil portfolio using extreme value theory and vine copula. Physica A: Statistical Mechanics and its Applications, 490, 1423-1433.

[65] Zhang, B., Wei, Y., Yu, J., Lai, X., \& Peng, Z. (2014). Forecasting VaR and ES of stock index portfolio: a Vine copula method. Physica A: Statistical Mechanics and its Applications, 416, 112-124.

[66] Zhang, T., \& Liu, Z. (2017). Fireworks algorithm for mean-VaR/CVaR models. Physica A: Statistical Mechanics and its Applications, 483, 1-8.

[67] Zhao, S., Lu, Q., Han, L., Liu, Y., \& Hu, F. (2015). A mean-CVaR-skewness portfolio optimization model based on asymmetric Laplace distribution. Annals of Operations Research, 226(1), 727-739. 
[68] Zhou, W. X. (2009). The components of empirical multifractality in financial returns. EPL (Europhysics Letters), 88(2), 28004.

\section{Appendix}

The $L p_{\text {min }}$ method (Giacalone, 1997; Giacalone \& Richiusa, 2006) is based on a two-steps alternating procedure: a) minimization procedure to estimate the parameters; b) joint inverse function of $I$ in (3) and $\beta_{2}$ in (2) to estimate $p$.

The method estimates the $p$ parameter considering the minimum between two functions, respectively given by the difference between empirical and theoretical $I$, and empirical and theoretical $\beta_{2}$. As the two kurtosis indexes have different orders of magnitude and different variance, the two differences are divided by the maximum theoretical value obtainable: by considering $p$ as ranging from 0.5 to 10, then 25.2 is the maximum value of $\beta_{2}$, and 0.86054 is the maximum value of $I$ (see Table 1 ). In this way, it is possible to obtain a joint estimator made up of two squared functions. The two kurtosis indexes are chosen as it was observed that norm-1 kurtosis ( $I$ index) is a valid choice for the estimation of $p$ in presence of outliers, while norm- 2 kurtosis ( $\beta_{2}$ index) performs better when the sample values gather around the center of the distribution.

When the value of $p$ is known, the estimates of $\mu$ and $\sigma_{p}$ are equal for both $L p$-norm and Maximum Likelihood estimators (Giacalone, 1997). Specifically, the function to be minimized to estimate $p$ is:

$$
p \mapsto\left[\frac{I-\hat{I}}{0.86054}\right]^{2}+\left[\frac{\beta_{2}-\hat{\beta_{2}}}{25.2}\right]^{2},
$$

where $I, \hat{I}, \beta_{2}, \hat{\beta}_{2}$ are given by (3), (2) and (4).

Tables 2-4 show a comparison of mean, variance, and mean squared error of three different empirical distributions of $p(n=50, n=100$, and $n=200)$, obtained by means of three estimation methods: the $L p_{g m}$ method by Gonin and Money (1987), the Agrò's (1995) $L p_{+}$method, and the $L p_{\min }$ method proposed in this paper.

For $p=1.2$ and $p=1.5$ (leptokurtic distributions), $L p_{\min }$ appears to be the most efficient method, taking both variance and mean squared error into account. In general, the method presents a trend which is similar to that of $L p_{+}$with regard to the accuracy of estimates, but with a gain in efficiency. For $p=2$, the $L p_{g m}$ method seems preferable, since, for any theoretical value of $p$, it provides estimates that gravitate around the value $p=2$. For $p=2.5, p=3.0$, and $p=3.5$ (platykurtic distributions), the $L p_{g m}$ method presents lower values of variance and mean squared error, compared to $L p_{+}$and $L p_{\min }$. Nonetheless, once the means in Tables $2-4$ are compared, the $L p_{g m}$ method presents a growing level of distortion as $n$ increases, suggesting that it is not superior to the other considered methods. Indeed, the most interesting aspect to be highlighted is the different asymptotic behavior of the three methods: asymptotic accuracy is a very desirable property of 


\begin{tabular}{|c|c|c|c|c|c|c|}
\hline$p$ & 1.2 & 1.5 & 2.0 & 2.5 & 3.0 & 3.5 \\
\hline$M\left(p_{g m}\right)$ & 1.5349 & 1.8927 & 2.2161 & 2.5012 & 2.6938 & 2.8734 \\
\hline$V\left(p_{g m}\right)$ & 0.1097 & 0.1546 & 0.2236 & 0.2479 & 0.2566 & 0.2637 \\
\hline$M S E\left(p_{g m}\right)$ & 0.2219 & 0.3088 & 0.2703 & 0.2479 & 0.3504 & 0.6563 \\
\hline$M\left(p_{+}\right)$ & 1.6957 & 1.9985 & 2.5561 & 3.0392 & 3.5245 & 4.0822 \\
\hline$V\left(p_{+}\right)$ & 0.3515 & 0.4846 & 1.8152 & 2.1054 & 2.4296 & 3.1064 \\
\hline$M S E\left(p_{+}\right)$ & 0.5972 & 0.7331 & 2.1244 & 2.3961 & 2.7047 & 3.4453 \\
\hline$M\left(p_{\min }\right)$ & 1.4374 & 1.7485 & 2.2656 & 2.7222 & 3.2114 & 3.4099 \\
\hline$V\left(p_{\min }\right)$ & 0.1221 & 0.3577 & 0.6451 & 1.0566 & 1.4437 & 1.6687 \\
\hline$M S E\left(p_{\min }\right)$ & 0.1784 & 0.4135 & 0.7156 & 1.1060 & 1.4884 & 1.6768 \\
\hline
\end{tabular}

Table 2: Mean (M), Variance (V), and Mean Squared Error (MSE) of $p$, estimated through different methods, with sample size $n=50$.

estimators, and no other merit assessment may compensate for the absence of such property. While $L p_{+}$and $L p_{\min }$ seem to guarantee asymptotically accurate estimates, $L p_{g m}$ shows an increase in the estimates' distortion when moving on from smaller to bigger sample sizes. From an asymptotic point of view, we can say that the $L p_{\min }$ method seems more valid in the $p<2$ case, while the $L p_{+}$ method seems preferable in the $p>2$ case. 


\begin{tabular}{|c|c|c|c|c|c|c|}
\hline$p$ & 1.2 & 1.5 & 2.0 & 2.5 & 3.0 & 3.5 \\
\hline$M\left(p_{g m}\right)$ & 1.4924 & 1.7850 & 2.1361 & 2.3298 & 2.6245 & 2.8734 \\
\hline$V\left(p_{g m}\right)$ & 0.0762 & 0.0841 & 0.1052 & 0.1054 & 0.1296 & 0.2637 \\
\hline$M S E\left(p_{g m}\right)$ & 0.1617 & 0.1653 & 0.1237 & 0.1177 & 0.2706 & 0.6563 \\
\hline$M\left(p_{+}\right)$ & 1.4023 & 1.8087 & 2.2302 & 2.6892 & 3.1675 & 3.6786 \\
\hline$V\left(p_{+}\right)$ & 0.0354 & 0.0691 & 0.3419 & 0.5591 & 0.9547 & 1.1492 \\
\hline$M S E\left(p_{+}\right)$ & 0.0763 & 0.1613 & 0.3949 & 0.5948 & 0.9827 & 1.1811 \\
\hline$M\left(p_{\min }\right)$ & 1.3451 & 1.6206 & 2.1228 & 2.5760 & 3.1464 & 3.5136 \\
\hline$V\left(p_{\min }\right)$ & 0.0515 & 0.1188 & 0.2535 & 0.4365 & 0.8296 & 1.0814 \\
\hline$M S E\left(p_{\min }\right)$ & 0.0725 & 0.1333 & 0.2688 & 0.4423 & 0.8510 & 1.0815 \\
\hline
\end{tabular}

Table 3: Mean (M), Variance (V), and Mean Squared Error (MSE) of $p$, estimated through different methods, with sample size $n=100$ 


\begin{tabular}{lcccccc}
$p$ & 1.2 & 1.5 & 2.0 & 2.5 & 3.0 & 3.5 \\
\hline$M\left(p_{\text {gm }}\right)$ & 1.5307 & 1.7143 & 2.0596 & 2.3458 & 2.5733 & 2.7622 \\
$V\left(p_{g m}\right)$ & 0.0207 & 0.0446 & 0.0559 & 0.0589 & 0.0514 & 0.0585 \\
& & & & & & \\
$M S E\left(p_{g m}\right)$ & 0.1301 & 0.0905 & 0.0594 & 0.0827 & 0.2335 & 0.6028 \\
\hline & & & & & & \\
\hline$M\left(p_{+}\right)$ & 1.4157 & 1.7386 & 2.0861 & 2.5182 & 2.9188 & 3.3094 \\
& & & & & & \\
$V\left(p_{+}\right)$ & 0.0495 & 0.0221 & 0.0624 & 0.1608 & 0.2747 & 0.3564 \\
$M S E\left(p_{+}\right)$ & 0.0960 & 0.0790 & 0.0698 & 0.1611 & 0.2813 & 0.3927 \\
\hline & & & & & & \\
\hline$M\left(p_{\text {min }}\right)$ & 1.2784 & 1.5553 & 2.0641 & 2.5311 & 3.0423 & 3.5289 \\
$V\left(p_{\text {min }}\right)$ & 0.0348 & 0.0476 & 0.0975 & 0.2741 & 0.4996 & 0.6135 \\
$M S E\left(p_{\text {min }}\right)$ & 0.0411 & 0.0507 & 0.1016 & 0.2751 & 0.5014 & 0.6143
\end{tabular}

Table 4: Mean (M), Variance (V), and Mean Squared Error (MSE) of $p$, estimated through different methods, with sample size $n=200$ 